H. Kang and J. K. Seo

Nagoya Math. J.

Vol. 138 (1995), 19-32

\title{
CAUCHY TRANSFORMS ON POLYNOMIAL CURVES AND RELATED OPERATORS
}

\author{
HYEONBAE KANG AND JIN KEUN SEO
}

\section{Introduction and statement of results}

Let $\Gamma$ be a curve in $\mathbf{R}^{2}$ defined by $y=A(x)$. The Cauchy transform $\mathscr{C}_{A}$ on $\Gamma$ is defined by the kernel

$$
K(x, y)=\frac{1+i A(y)}{(x-y)+i(A(x)-A(y))} .
$$

When $A$ is a Lipschitz function, the $L^{2}$ boundedness of $\mathscr{C}_{A}$ is well understood and several proofs of it have been produced (cf. [C, CJS, CMM, DJ, M]). If $A$ is a $C^{1}$-smooth function, then the local $L^{2}$ boundedness of $\mathscr{C}_{A}$ is also well understood (cf. [FJR]). However, if $A$ is a smooth, not necessarily Lipschitz function, the question of global $L^{2}$ boundedness of $\mathscr{C}_{A}$ has not been settled. In [KS], we observe that $\mathscr{C}_{A}$ is not, in general, bounded on $L^{2}$ if $A$ is a smooth non-Lipschitz function, and prove that $\mathscr{C}_{A}$ is bounded on $L^{2}$ if $A$ is either a polynomial of odd degree or an even polynomial. The purpose of this paper is to give a new proof of it and to extend the result to arbitrary polynomials.

THEOREM. If $A$ is a polynomial, then the Cauchy transform on the curve $y=$ $A(x)$ is bounded on $L^{p}(\mathbf{R}), 1<p<\infty$.

In [KS], we used a continuously varying cut-off function to separate the singularities of $K(x, y)$ at $x=y$ and at $x=-y$ and the T1-theorem of David and Journé. In this paper, instead of using a cut-off function, we use a direct decomposition of the Cauchy kernel. If $A$ is a polynomial, then the Cauchy kernel $K(x, y)$ can be decomposed as

Received March 28, 1994.

This work was supported in part by GARC-KOSEF and NON-DIRECTED RESEARCH FUND, Korea Research Foundation, 1993. 


$$
K(x, y)=\frac{1+i A(y)}{(x-y)+i(A(x)-A(y))}=\frac{1}{x-y}+i \frac{P(x, y)}{1+i Q(x, y)}
$$

where

$$
Q(x, y)=\frac{A(x)-A(y)}{x-y} \text { and } P(x, y)=\frac{A^{\prime}(y)-Q(x, y)}{x-y}
$$

Moreover, if $A$ is an even polynomial, one can easily see that $Q(x, y)=$ $(x+y) R(x, y)$ for some polynomial $R$. Define an operator $T_{A}$ by

$$
T_{A} f(x)=\int_{-\infty}^{\infty} \frac{P(x, y)}{1+i Q(x, y)} f(y) d y .
$$

Then, $\mathscr{C}_{A}=\mathscr{H}+i T_{A}$ where $\mathscr{H}$ is the Hilbert transform. We prove that $T_{A}$ is bounded on $L^{p}(\mathbf{R}), 1<p<\infty$. If $A$ is a polynomial of odd degree, it is easy to prove the $L^{2}$ boundedness of $T_{A}$ since $Q(x, y)$ does not have any zero when $x^{2}+$ $y^{2}$ is large. If $A$ is an even polynomial, then we compare $T_{A}$ with a linear combination of the Hilbert transform and various operators defined in Section 3 . We show that these operators are bounded on $L^{p}(\mathbf{R})$ and that the difference of $T_{A}$ and a linear combination of the Hilbert transform and these operators can be estimated by the Hardy Littlewood maximal operator which is well known to be bounded on $L^{p}$ (cf. [S]). If $A$ is a polynomial of even degree, then there exists a change of variables $\alpha(x)$ defined for large $x$ such that $A(\alpha(x))$ becomes an even polynomial. By carefully studying the behavior of $\alpha(x)$ for large $x$, we are able to reduce matters to the case of even polynomials.

We organize this paper as follows; in Section 2, we prove some properties of $Q(x, y)$ which will be used in later sections. In Section 3 , we introduce some related operators and prove that they are bounded on $L^{p}(\mathbf{R})$. In Section 4 , we prove that $T_{A}$ is bounded on $L^{p}(\mathbf{R})$ if $A$ is a polynomial of odd degree. In the final section, we prove that $T_{A}$ is bounded on $L^{p}(\mathbf{R})$ if $A$ is a polynomial of even degree.

We use a standard notation of $A \leqslant B$ to imply that $A \leq C B$ for some constant $C . A \approx B$ means that both $A \lesssim B$ and $A \gtrsim B$ hold.

\section{Preliminary on polynomials}

Let $A$ be a polynomial and let

$$
Q(x, y)=\frac{A(x)-A(y)}{x-y}
$$


In this section, we collect some properties of $Q$ which will be used in later sections.

LEMMA 2.1.

(1) If $\operatorname{deg} A=2 n+1$, then there exists a positive constant $r$ such that

$$
|Q(x, y)| \geq x^{2 n}+y^{2 n}
$$

if $x^{2}+y^{2} \geq r$.

(2) If $A$ is an even polynomial and $\operatorname{deg} A=2 n+2$, then there exist a positive constant $r$ and a polynomial $R(x, y)$ such that

$$
Q(x, y)=(x+y) R(x, y)
$$

and

$$
|R(x, y)| \geq x^{2 n}+y^{2 n}
$$

if $x^{2}+y^{2} \geq r$.

Proof. For (1), note that

$$
\begin{aligned}
\frac{x^{2 n+1}-y^{2 n+1}}{x-y} & =\sum_{j=0}^{2 n} x^{2 n-j} y^{j} \\
& =\frac{1}{2}\left(x^{2 n}+y^{2 n}\right)+\frac{1}{2}(x+y)^{2} \sum_{j=1}^{n} x^{2(n-j)} y^{2(j-1)} \\
& \geq \frac{1}{2}\left(x^{2 n}+y^{2 n}\right) .
\end{aligned}
$$

Let $A(x)=\sum_{\jmath=0}^{2 n+1} a_{j} x^{\jmath}\left(a_{2 n+1} \neq 0\right)$. Then

$$
\begin{aligned}
\left|\frac{A(x)-A(y)}{x-y}\right| & \geq \frac{\left|a_{2 n+1}\right|}{2}\left(x^{2 n}+y^{2 n}\right)-\sum_{j=1}^{2 n-1} j\left|a_{j}\right|\left(|x|^{j}+|y|^{j}\right) \\
& \geq x^{2 n}+y^{2 n}
\end{aligned}
$$

if $x^{2}+y^{2}$ is large.

For (2), we note that

$$
\begin{aligned}
\left|x^{2 j}-y^{2 j}\right| & =|x-y||x+y|\left|x^{2 j-2}+x^{2 j-4} y^{2}+\cdots+x^{2} y^{2 j-4}+y^{2 j-2}\right| \\
& \approx|x-y||x+y|\left(|x|^{2 j-2}+|y|^{2 j-2}\right) .
\end{aligned}
$$

Let $A(x)=\sum_{j=0}^{2 n+2} a_{j} x^{2 j}\left(a_{2 n+1} \neq 0\right)$. Then 


$$
\begin{aligned}
|A(x)-A(y)| & \geq\left|a_{2 n+1}\right|\left|x^{2 n+2}+y^{2 n+2}\right|-\sum_{j=1}^{2 n}\left|a_{j}\right|\left|x^{2 j}-y^{2 j}\right| \\
& \geq|x-y||x+y|\left[\left|a_{2 n+2}\right|\left(|x|^{2 n}+|y|^{2 n}\right)-C\left(|x|^{2 n-2}+|y|^{2 n-2}+1\right)\right] \\
& \geq|x-y||x+y|\left(|x|^{2 n}+|y|^{2 n}\right)
\end{aligned}
$$

for some constants $C$ as long as $x^{2}+y^{2}$ is large. This completes the proof.

The next lemma and corollary show that a polynomial of even degree is essentially the same as an even polynomial for our purpose.

Lemma 2.2. Let $A$ be a polynomial of even degree. Then, there exist $r>0$ and a smooth change of variable $\alpha(x)$ on $|x|>r$ such that

(1) $A(\alpha(x))$ is an even polynomial,

(2) $\alpha(x)=x+\beta(x)$ where $\beta(x)=O(1)$ and $\beta^{\prime}(x)=O(1 / x)$ as $x \rightarrow \infty$.

Proof. Let $A(x)=\sum_{j=0}^{2 n} a_{j} x^{\prime}$ and assume that $a_{2 n}=1$ without loss of generality. Choose $r>0$ so that $A$ is monotone if $|x|>r$ and define $\alpha$ on $|x|>r$ by

$$
A(\alpha(x))=\frac{A(x)+A(-x)}{2} .
$$

Since $A(x) \approx x^{2 n}$ and $A^{\prime}(x) \approx x^{2 n-1}$ if $|x|>r$ by increasing $r$ if necessary, one can easily see that $\alpha(x) \approx x$ and $\alpha^{\prime}(x) \approx 1$. From (2.1), we have

$$
\alpha(x)^{2 n}+\sum_{j=0}^{2 n-1} a_{j} \alpha(x)^{j}=x^{2 n}+\sum_{j=0}^{n-1} a_{2 j} x^{2 j} .
$$

It then follows that

$$
\alpha(x)^{2 n}=x^{2 n}+O\left(x^{2 n-1}\right)
$$

and

$$
2 n \alpha(x)^{2 n-1} \alpha^{\prime}(x)=2 n x^{2 n-1}+O\left(x^{2 n-2}\right) .
$$

It follows immediately from these relations that

$$
\alpha(x)=x+O(1) \text { and } \alpha^{\prime}(x)=1+O\left(\frac{1}{x}\right) \text { as } x \rightarrow \infty .
$$

This completes the proof. 
COROllary 2.3. Let $\beta$ and $r$ be as above. Then,

$$
\left|\frac{\beta(x)-\beta(y)}{x-y}\right| \leqslant \frac{1}{|x|+|y|}
$$

if $x^{2}+y^{2} \geq r$.

Proof. If $x y<0$, then there is nothing to prove. Suppose that $x y>0$ and that $y>x>0$ without loss of generality. If $y>2 x$, then since $\beta(x)=O(1)$ as $x$ $\rightarrow \infty$, we have

$$
\left|\frac{\beta(x)-\beta(y)}{x-y}\right| \leq \frac{1}{y} \leq \frac{1}{|x|+|y|} .
$$

If $x<y<2 x$, then since $\beta^{\prime}(x)=O(1 / x)$ as $x \rightarrow \infty$, we have

$$
\left|\frac{\beta(x)-\beta(y)}{x-y}\right|=\left|\beta^{\prime}(\xi)\right| \leq \frac{1}{\xi} \leq \frac{1}{x} \leqslant \frac{1}{|x|+|y|}
$$

for some $x<\xi<y$. This completes the proof.

\section{Related operators}

In this section we introduce some related operators and show that they are bounded on $L^{p}(\mathbf{R})$ by comparing them with the Hardy Littlewood maximal operator and the Hilbert transform. Throughout this paper $M$ denotes the Hardy Littlewood maximal operator.

Proposition 3.1. Let $P(x, y)$ and $R(x, y)$ be smooth functions such that there exists a positive constant $r$ so that

$$
|P(x, y)| \leq|x|^{n}+|y|^{n} \text { and }|R(x, y)| \geq|x|^{n}+|y|^{n} \text { if } x^{2}+y^{2} \geq r \text {. }
$$

Suppose that $0 \leq \alpha$ and $0<\beta-\alpha \leq \gamma$. For $f \in C_{0}^{\infty}(\mathbf{R})$, define

$$
U f(x)=\int_{-\infty}^{\infty} \frac{|x-y|^{\alpha}|P(x, y)|}{1+|x-y|^{\beta}|R(x, y)|^{\gamma}}|f(y)| d y .
$$

If $\gamma \geq 1+1 / n$, then

$$
|U f(x)| \gtrsim M f(x)
$$

for every $x$. 
Proof. For simplicity, we assume that $r=1$. Write

$$
U f(x)=\int_{|x-y| \leq 1}+\int_{|x-y|>1}:=\mathrm{I}+\mathrm{II} .
$$

Since $0<\beta-\alpha$ and $\gamma \geq 1+1 / n$, we have

$$
\begin{aligned}
|\mathrm{II}| \lesssim & \int_{|x-y|>1} \frac{|x-y|^{\alpha}(|x|+|y|)^{n}}{1+|x-y|^{\beta}(|x|+|y|)^{\gamma n}}|f(y)| d y \\
\lessgtr & \int_{1<|x-y| \leq 1+|x|} \frac{(1+|x|)^{n}}{1+|x|^{\gamma n}}|f(y)| d y \\
& \quad+\sum_{j=1}^{\infty} \int_{2^{j-1}(1+|x|)<|x-y| \leq 2^{j}(1+|x|)} \frac{2^{n \jmath}(1+|x|)^{n}}{2^{(\beta-\alpha+\gamma n) j}(1+|x|)^{\beta-\alpha+\gamma n}}|f(y)| d y \\
\lesssim & \left(1+\sum_{j=1}^{\infty} 2^{(1+n-\beta+\alpha-\gamma n) j}(1+|x|)^{1+n-\beta+\alpha-\gamma n}\right) M f(x) \\
\lesssim & M f(x) .
\end{aligned}
$$

We now deal with I. If $|x| \leq 1$, then it is obvious that $|\mathrm{I}| \leq M f(x)$. Suppose that $|x| \geq 1$. Then

$$
\begin{aligned}
|\mathrm{I}| & \leq \sum_{j=1}^{\infty} \int_{2^{-j}<|x-y|<2^{-j+1}} \frac{2^{-\alpha j}\left(|x|+|y|^{n}\right)}{1+2^{-\beta j}\left(|x|^{\gamma n}+|y|^{\gamma n}\right)}|f(y)| d y \\
& \leq \sum_{j=1}^{\infty} \frac{|x|^{n} 2^{-(\alpha+1) j}}{1+2^{-\beta j}|x|^{\gamma n}} M f(x) .
\end{aligned}
$$

Pick $N$ so that $1 \leq|x|^{n} 2^{-N} \leq 10$. Then we obtain

$$
\sum_{j=N}^{\infty} \frac{|x|^{n} 2^{-(\alpha+1) \jmath}}{1+2^{-\beta j}|x|^{r n}} \leq|x|^{n} 2^{-N+1} \leq 20
$$

We also obtain

$$
\sum_{j=1}^{N} \frac{|x|^{n} 2^{-(\alpha+1) j}}{1+2^{-\beta j}|x|^{\gamma n}} \leq \sum_{j=N}^{N} 2^{(\beta-\alpha-1) j}|x|^{(1-\gamma) n} \leq 2^{-(\gamma-1) N} \sum_{j=1}^{N} 2^{(\beta-\alpha-1) j} \leq C
$$

because $|x|^{(1-\gamma) n} \leq 2^{-(\gamma-1) N}$ and $\beta-\alpha \leq \gamma$. This completes the proof.

Remark 3.2. The condition on $\gamma$ in Proposition 3.1 are sharp in the sense that if $\gamma<1+1 / n$, then (3.2) does not hold. 
Proposition 3.3. Let $P(x, y)$ be a homogeneous polynomial of degree $2 n-1$. Let

$$
V f(x)=\int_{-\infty}^{\infty} \frac{|P(x, y)|}{x^{2 n}+y^{2 n}}|f(y)| d y
$$

for $f \in C_{0}^{\infty}(\mathbf{R})$. Then, $V$ extends to be an operator bounded on $L^{p}(\mathbf{R}), 1<p<\infty$.

Proof. Let $f \in C_{0}^{\infty}(\mathbf{R})$. Let

$$
V_{1} f(x)=\int_{-\infty}^{\infty} \frac{|x|^{2 n-1}}{x^{2 n}+y^{2 n}}|f(y)| d y .
$$

We will show that

$$
\left|V_{1} f(x)\right| \lesssim M f(x)
$$

Assume $x \neq 0$.

$$
\begin{aligned}
\left|V_{1} f(x)\right| & \leq|x|^{2 n-1}\left(\int_{|y| \leq 2|x|}+\sum_{j=1}^{\infty} \int_{2^{j-1}|x|<|x-y| \leq 2^{j}|x|} \frac{1}{x^{2 n}+y^{2 n}}|f(y)| d y\right) \\
& \leq|x|^{2 n-1}\left(\int_{|x-y| \leq 3|x|} x^{-2 n}|f(y)| d y+\sum_{j=1}^{\infty} \int_{|x-y| \leq 2^{j}|x|}\left(2^{j} x\right)^{-2 n}|f(y)| d y\right) \\
& \leqslant\left(1+\sum_{j=1}^{\infty} 2^{j(1-2 n)}\right) M f(x) \leqslant M f(x) .
\end{aligned}
$$

Hence $V_{1}$ extends to be an operator bounded on $L^{p}(\mathbf{R}), 1<p<\infty$.

Since $|P(x, y)| \lesssim|x|^{2 n-1}+|y|^{2 n-1}$, we have

$$
|V f| \lesssim V_{1} f+V_{1}^{*} f
$$

where $V_{1}^{*}$ is the adjoint of $V_{1}$. Hence Proposition 3.3 follows from $L^{p}$-boundedness of $V_{1}$ and $V_{1}^{*}$. This completes the proof.

Corollary 3.4. Let $P(x, y)$ and $R(x, y)$ be homogeneous polynomials of degree 2n. Assume that

$$
|R(x, y)| \geq x^{2 n}+y^{2 n}
$$

For $f \in C_{0}^{\infty}(\mathbf{R})$, define

$$
W f(x)=\int_{-\infty}^{\infty} \frac{P(x, y)}{(x-y) R(x, y)} f(y) d y .
$$


Then, $W$ extends to be an operator bounded on $L^{p}(\mathbf{R}), 1<p<\infty$.

Proof. Let $a=P(x, x) / R(x, x)$. Then, we have

$$
\frac{P(x, y)}{(x-y) R(x, y)}-\frac{a}{x-y}=\frac{P(x, y)-a R(x, y)}{x-y} \frac{1}{R(x, y)} .
$$

Note that $(P(x, y)-a R(x, y)) /(x-y)$ is a homogeneous polynomial of degree $2 n-1$. Hence Corollary 3.4 follows from the $L^{p}$-boundedness of the Hilbert transform and Proposition 3.3. This completes the proof.

\section{Cauchy transform I}

Recall that $T_{A}$ is the operator defined by the kernel

$$
\frac{P(x, y)}{1+i Q(x, y)}
$$

where

$$
Q(x, y)=\frac{A(x)-A(y)}{x-y} \text { and } P(x, y)=\frac{A^{\prime}(y)-Q(x, y)}{x-y}
$$

and that

$$
\mathscr{C}_{A}=\mathscr{H}+i T_{A}
$$

where $\mathscr{H}$ is the Hilbert transform. We now prove that the operator $T_{A}$ is bounded on $L^{p}(\mathbf{R})$. We first deal with the case when $\operatorname{deg} A$ is odd in this section.

Theorem 4.1. Let $P(x, y)$ and $Q(x, y)$ be polynomials of degree $2 n-1$ and $2 n$, respectively. Assume that there exists a constant $r$ such that

$$
\begin{aligned}
& \qquad|Q(x, y)| \geq x^{2 n}+y^{2 n} \\
& \text { if } x^{2}+y^{2} \geq r \text {. For } f \in C_{0}^{\infty}(\mathbf{R}) \text {, define } \\
& T f(x)=\int_{-\infty}^{\infty} \frac{P(x, y)}{1+i Q(x, y)} f(y) d y .
\end{aligned}
$$

Then, $T$ extends to be an operator bounded on $L^{p}(\mathbf{R}), 1<p<\infty$.

Proof. Let $q$ be the conjugate of $p$, and let 


$$
k(x, y)=\frac{P(x, y)}{1+i Q(x, y)} .
$$

Then

$$
\int|T f(x)|^{p} d x \leqslant \int_{|x| \leq r}|T f(x)|^{p} d x+\int_{|x|>r}|T f(x)|^{p} d x:=\mathrm{I}+\mathrm{II} .
$$

If $|x| \leq r$, then

$$
\int_{-\infty}^{\infty}|k(x, y)|^{q} d y \leq 1+\int_{|y|>r} \frac{y^{(2 n-1) q}}{1+y^{2 q n}} d y \leq C .
$$

It then follows from the Hölder inequality that

$$
I \leq \int_{|x| \leq r}\left(\int|k(x, y)|^{q} d y\right)^{p / q} d x\|f\|_{p}^{p} \leq\|f\|_{p}^{p} .
$$

For II, observe that if $|x|>r$, then

$$
|T f(x)| \leqslant \int_{-\infty}^{\infty} \frac{|x|^{2 n-1}+|y|^{2 n-1}}{x^{2 n}+y^{2 n}}|f(y)| d y .
$$

Hence, it follows from the proof of Proposition 3.3 that

$$
\int_{|x|>r}|T f(x)|^{p} d x \leqslant\|f\|_{p}^{p}
$$

This completes the proof.

COROLlary 4.2. If $A$ is a polynomial of odd degree, then the Cauchy transform $\mathscr{C}_{A}$ is bounded on $L^{p}(\mathbf{R}), 1<p<\infty$.

Proof. It follows from Theorem 4.1 and Lemma 2.1.

\section{Cauchy transform II}

In this section we prove that if $A$ is a polynomial of even degree, then the operator $T_{A}$ is bounded on $L^{p}$. We first deal with the case when $A$ is an even polynomial.

Theorem 5.1. Suppose that $P(x, y)=P_{0}(x, y)+P_{1}(x, y)$ and $R(x, y)=$ $R_{0}(x, y)+R_{1}(x, y)$ satisfy the following 
(1) $P_{0}(x, y)$ and $R_{0}(x, y)$ are homogeneous polynomial of degree $2 n$,

(2) $\left|P_{1}(x, y)\right| \leqslant|x|^{2 n-1}+|y|^{2 n-1}$ and $\left|R_{1}(x, y)\right| \leq|x|^{2 n-1}+|y|^{2 n-1}$ if $x^{2}$ $+y^{2}>r$ for some $r$,

(3) $|R(x, y)| \geq|x|^{2 n}+|y|^{2 n}$ if $x^{2}+y^{2}>r$.

For $f \in C_{0}^{\infty}(\mathbf{R})$, define

$$
T f(x)=\int_{-\infty}^{\infty} \frac{P(x, y)}{1+i(x-y) R(x, y)} f(y) d y .
$$

Then, $T$ extends to be an operator bounded on $L^{p}(\mathbf{R}), 1<p<\infty$.

Proof. By a straightforward computation, we have

$$
\begin{aligned}
& \left|\frac{P(x, y)}{1+i(x-y) R(x, y)}-\frac{P_{0}(x, y)}{1+i(x-y) R_{0}(x, y)}\right| \\
& \quad \leq \frac{\left|P(x, y)-P_{0}(x, y)\right|}{1+|x-y|^{2}\left|R_{0}(x, y) R(x, y)\right|}+\frac{\left|R_{0}(x, y) P(x, y)-P_{0}(x, y) R(x, y)\right|}{1+|x-y|\left|R_{0}(x, y) R(x, y)\right|} .
\end{aligned}
$$

Note that $\left|P(x, y)-P_{0}(x, y)\right| \leqslant|x|^{2 n}+|y|^{2 n},\left|R_{0}(x, y) P(x, y)-P_{0}(x, y) R(x, y)\right|$ $\lesssim|x|^{4 n-1}+|y|^{4 n-1}$, and $\left|R_{0}(x, y) R(x, y)\right| \gtrsim|x|^{4 n}+|y|^{4 n}$ if $|x|+|y|$ is large. It follows from Proposition 3.1 that

$$
\int_{-\infty}^{\infty}\left|\frac{P(x, y)}{1+i(x-y) R(x, y)}-\frac{P_{0}(x, y)}{1+i(x-y) R_{0}(x, y)}\right||f(y)| d y \leqslant M f(x) .
$$

Hence we may assume $P(x, y)$ and $R(x, y)$ are homogeneous polynomials of degree $2 n$. Note that

$$
\frac{P(x, y)}{1+i(x-y) R(x, y)}=\frac{P(x, y)}{1+(x-y)^{2} R(x, y)^{2}}+i \frac{(x-y) R(x, y) P(x, y)}{1+(x-y)^{2} R(x, y)^{2}} .
$$

The operator defined by the first kernel on the right hand side is proved to be bounded on $L^{p}(\mathbf{R})$ in Proposition 3.1. Let

$$
T_{1} f(x)=\int_{-\infty}^{\infty} \frac{(x-y) R(x, y) P(x, y)}{1+(x-y)^{2} R(x, y)^{2}} f(y) d y
$$

We compare $T_{1}$ with the operator $W$ defined in Corollary 3.4 with the same $P$ and $R$. Let $T_{2} f(x)=T_{1} f(x)-W f(x)$. Then

$$
T_{2} f(x)=\int_{-\infty}^{\infty} \frac{P(x, y)}{(x-y) R(x, y)\left(1+(x-y)^{2} R(x, y)^{2}\right)} f(y) d y
$$


With $a=P(x, x) / R(x, x)$, define

$$
T_{3} f(x)=T_{2} f(x)-a \mathscr{H} f(x)
$$

where $\mathscr{H}$ is the Hilbert transform. Then

$$
\begin{aligned}
& \frac{1}{x-y}\left(\frac{P(x, y)}{R(x, y)\left(1+(x-y)^{2} R(x, y)^{2}\right)}-a\right) \\
& =\frac{E(x, y)}{R(x, y)\left(1+(x-y)^{2} R(x, y)^{2}\right)}-a \frac{(x-y) R^{2}(x, y)}{1+(x-y)^{2} R^{2}(x, y)}
\end{aligned}
$$

where $E(x, y)=(P(x, y)-a R(x, y)) /(x-y)$ is a polynomial of degree $2 n-1$. Hence, by Proposition 3.3, it suffices to show $L^{p}$-boundedness of the operator $T_{4}$ defined by

$$
T_{4} f(x)=\int_{-\infty}^{\infty} \frac{(x-y) R^{2}(x, y)}{1+(x-y)^{2} R^{2}(x, y)} f(y) d y .
$$

Let $\varphi(x)=|x|^{-2 n}$ if $|x| \geq 1$ and $\varphi(x)=1$ if $|x|<1$. By similar estimates as above and Proposition 3.1, we obtain

$$
\left|T_{4} f(x)-\int_{|x-y| \geq \varphi(x)} \frac{f(y)}{x-y} d y\right| \lesssim M f(x) .
$$

Note that

$$
\left|\int_{|x-y| \geq \varphi(x)} \frac{f(y)}{x-y} d y\right| \lesssim \sup _{\varepsilon>0}\left|\int_{|x-y| \geq \varepsilon} \frac{f(y)}{x-y} d y\right|
$$

Since the right hand side is bounded on $L^{2}$ (cf. p. 42 , [S]), the proof is completed.

Corollary 5.2. If $A$ is an even polynomial, then the Cauchy transform $\mathscr{C}_{A}$ is bounded on $L^{p}(\mathbf{R}), 1<p<\infty$.

Proof. It follows from Theorem 5.1 and Lemma 2.1.

We now deal with the case when $A$ is a polynomial of even degree.

Theorem 5.3. Let $A$ be a polynomial of even degree. Then, the operator $T_{A}$ is bounded on $L^{p}(\mathbf{R}), 1<p<\infty$.

Proof. Let $A(x)=\sum_{j=0}^{2 n+2} a_{j} x^{j}$ and let $r$ be the number given in Lemma 2.2. It 
suffices to prove

$$
\mathrm{I}:=\int_{|x|>r}\left|\int_{|y|>r} \frac{P(x, y)}{1+i Q(x, y)} f(y) d y\right|^{p} d x \leqslant\|f\|_{p}^{p} .
$$

In fact, the rest cases can be treated by the Hölder inequality since $|Q(x, y)| \approx$ $|x|^{2 n+1}+|y|^{2 n+1}$ if either $|x|<r$ and $|y|>2 r$, or $|x|>2 r$ and $|y|<r$. In order to estimate $\mathrm{I}$, we make changes of variables $y=\alpha(s)$ and $x=\alpha(t)$ defined in Lemma 2.2. Then, since $\alpha^{\prime}(s) \approx 1$, we have

$$
\mathrm{I} \lesssim \int_{-\infty}^{\infty}\left|\int_{-\infty}^{\infty} \frac{P(\alpha t), \alpha(s))}{1+i Q(\alpha(t), \alpha(s))} F(s) d s\right|^{p} d t
$$

where $F(s)=f(\alpha(s)) \alpha^{\prime}(s) \chi$ while $\chi$ is the characteristic function on $\{|\alpha(s)|>$ $r$. Let $B(t)=A(\alpha(t))$. Then, $B(t)$ is an even polynomial and

$$
Q(\alpha(t), \alpha(s))=\frac{B(t)-B(s)}{\alpha(t)-\alpha(s)} .
$$

Since $\alpha(t)=t+O(1)$, we have

$$
P(\alpha(t), \alpha(s))=P_{0}(t, s)+E(t, s)
$$

where $P_{0}(t, s)$ is a homogeneous polynomial of degree $2 n$ and $E(t, s)=O\left(|t|^{2 n-1}\right.$ $\left.+|s|^{2 n-1}\right)$ if $|t|+|s|$ is large. Since

$$
|Q(\alpha(t), \alpha(s))|=\left|\frac{B(t)-B(s)}{\alpha(t)-\alpha(s)}\right| \approx|t+s|\left(|t|^{2 n}+\mid s^{2 n}\right)
$$

for $|t|+|s|$ large, it is already proved in Proposition 3.1 that the operator defined by the kernel $E(t, s) /[1+i Q(\alpha(t), \alpha(s))]$ is bounded on $L^{p}$. For convenience, put

$$
k_{0}(t, s)=\frac{P_{0}(t, s)}{1+i Q(\alpha(t), \alpha(s))} \quad \text { and } \quad k(t, s)=\frac{P_{0}(t, s)}{1+i \frac{B(t)-B(s)}{t-s}} .
$$

Then, $k(t, s)$ defines an operator bounded on $L^{p}$ by Theorem 4.1. A straightforward computation gives

$$
k_{0}(t, s)-k(t, s)=\frac{i P_{0}(t, s) Q(\alpha(t), \alpha(s)) \frac{\beta(t)-\beta(s)}{t-s}}{[1+i Q(\alpha(t), \alpha(s))]\left[1+i \frac{B(t)-B(s)}{t-s}\right]}
$$


where $\alpha(t)=t+\beta(t)$ as defined in Lemma 2.1. It then follows from Corollary 2.3 that

$$
\begin{aligned}
\left|k_{0}(t, s)-k(t, s)\right| & \leq \frac{\left(|t|^{2 n}+|s|^{2 n}\right)\left(|t|^{2 n}+|s|^{2 n}\right)|t+s| \frac{1}{|t|+|s|}}{1+|t+s|^{2}\left(|t|^{2 n}+|s|^{2 n}\right)^{2}} \\
& \leq \frac{|t+s|\left(|t|^{4 n-1}+|s|^{4 n-1}\right)}{1+|t+s|^{2}\left(|t|^{2 n}+|s|^{2 n}\right)^{2}} .
\end{aligned}
$$

Hence, $\left|k_{0}(t, s)-k(t, s)\right|$ defines an operator bounded on $L^{p}$ by Proposition 3.1. This completes the proof.

Finally, we have the main theorem of this paper.

THEOREm. If $A$ is a polynomial, then the Cauchy transform on the curve $y=$ $A(x)$ is bounded on $L^{p}(\mathbf{R}), 1<p<\infty$.

\section{REFERENCES}

[C] A. P. Calderón, Cauchy integrals on Lipschitz curves and related operators, Proc. Nat. Acad. Sci. USA, 74 (1977), 1324-1327.

[CJS] R. R. Coifman, P. Jones, and S. Semmes, Two elementary proofs of the $L^{2}-$ boundedness of Cauchy integrals on Lipschitz curves, J. Amer. Math. Soc., 2 (1989), 553-564.

[CMM] R. R. Coifman, A. McIntosh, Y. Meyer, L'intégrale de Cauchy definit un opérateur bornée sur $L^{2}$ pour courbes lipschiziennes, Annals of Math., 116 (1982), 361-387.

[DJ] G. David and J.-L. Journé, A boundedness criterion for generalized CalderónZygmund operators, Annals of Math., 120 (1984), 371-397.

[FJR] E. B. Fabes, M. Jodeit Jr., and N. M. Riviére, Potential techniques for boundary value problems on $C^{1}$-domains, Acta Math., 141 (1978), 165-186.

[KS] H. Kang and J. K. Seo, $L^{2}$-boundedness of the Cauchy transform on smooth non-Lipschitz curves, Nagoya Math. J., 130 (1993), 123-147.

[M] T. Murai, A real variable method for the Cauchy transform, and analytic capac. ity, Lecture Note in Math., 1307, Springer-Verlag, New York, 1988.

[S] E. Stein, Singular Integrals and Differentiability Properties of Functions, Princeton Univ. Press Princeton, 1970.

Hyeonbae Kang

Department of Mathematics

Korea University

Seoul 136-701, Korea

e-mail:kang@kuccnx.korea.ac.kr 
Jin Keun Seo

Department of Mathematics POSTECH

P. O. Box 125, Pohang 790-600, Korea

e-mail:seoj@posmath.postech.ac.kr

Current address of J. K. Seo

Department of Mathematics

Yonsei University

Seovl 120-749, Korea

e-mail:seoj@bubble.yonsei.ac.kr 